\title{
In-Vivo Dosimetry Method for Measuring Peak Surface Dose Using Radiochromic Films during Computed Tomography Scanning of the Sinus
}

\author{
Khaled Soliman ${ }^{1}$, Salman Altimyat ${ }^{1}$, Abdullah Alrushoud ${ }^{1}$, \\ Ahmed Alenezi ${ }^{2}$, Mohammed Alkhorayef ${ }^{2}$ \\ ${ }^{1}$ Medical Physics Department, Prince Sultan Military Medical City, Riyadh, KSA \\ ${ }^{2}$ Department of Radiological Sciences, College of Applied Medical Sciences, King Saud University, Riyadh, KSA \\ Email: *khaledsoliman61@gmail.com
}

How to cite this paper: Soliman, K., Altimyat, S., Alrushoud, A., Alenezi, A. and Alkhorayef, M. (2018) In-Vivo Dosimetry Method for Measuring Peak Surface Dose Using Radiochromic Films during Computed Tomography Scanning of the Sinus. International Journal of Medical Physics, Clinical Engineering and Radiation Oncology, 7, 151-159.

https://doi.org/10.4236/ijmpcero.2018.72013

Received: March 8, 2018

Accepted: May 4, 2018

Published: May 7, 2018

Copyright () 2018 by authors and Scientific Research Publishing Inc. This work is licensed under the Creative Commons Attribution International License (CC BY 4.0).

http://creativecommons.org/licenses/by/4.0/ cc) (i) Open Access

\begin{abstract}
Purpose: During computed tomography (CT) helical scanning mode the patient surface dose distribution is assumed to be non-uniform, therefore point dose measurement methods may lead to imprecise estimation of the radiation dose received by the patient skin in particular. We have used XRQA2 films as in-vivo dosimeters to measure the entrance skin dose during sinus exams. Methods: The films were placed under the patient head rest in order to sample the entrance surface dose in-vivo. We have performed in-vivo film irradiation on 23 patients in this study to verify the clinical suitability of the method and were found adequate. Results: The measured average ESD in the sinus exam was $11.7 \pm 1.0 \mathrm{mGy}$, the PSD was $15.7 \pm 1.7 \mathrm{mGy}$ and the CTDI (vol) was

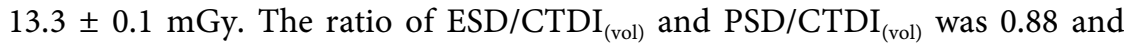
1.18 respectively. The results indicate that the scanner registered $\mathrm{CTDI}_{(\mathrm{vol})}$ underestimates the PSD and in the same time it overestimates the ESD by $18 \%$ and $13.6 \%$ respectively. Conclusion: The observed differences between the ESD, PSD and CTDI (vol) although seem small for the radiation dose range measured during CT of the sinus [13.2 - 13.4] mGy, but important for the medical physicist to know, since monitoring of patients' doses from CT examinations is becoming more mandatory. The use of radiochromic film as in-vivo dosimeter does not interfere with the clinical radiological exam and does not produce any image artifacts. The method can be used to study other CT examinations specially the ones with large beam width, high pitch factor and high dose exams. The method allows measurement of the peak skin dose, examination of the $\mathrm{CT}$ dose profile and the $2 \mathrm{D}$ dose distribution in the XZ plan.
\end{abstract}

\section{Keywords}

Radiochromic Films, Sinus CT, In-Vivo Dosimetry, $\mathrm{CTDI}_{(\mathrm{Vol})}$, Entrance 


\section{Introduction}

In the United States the estimated collective effective dose (CED) from CT examination constitutes $49 \%$ of the total CED from medical imaging procedures; and the annual number of procedures is about 67 million [1].

Radiation output of CT scanner must be tested, and equipment performance and patient dosimetry must be evaluated for each CT unit at least annually [2].

The American Association of Physicists in Medicine (AAPM) has recently published practice guidelines emphasizing clinical protocol review and management as an essential activity ensuring patient safety and is also part of the quality assurance (QA) program for CT [3]. It is recommended to monitor radiation doses from most common CT examinations performed in the institution. The sinus CT examination is one of the most common exams performed in our institution; there is an average of 5 exams performed daily.

In helical CT scanning with pitch factor of less than one like in this study there is X-ray beam overlap and the volume of tissue is irradiated more than once per scan. Such acquisition allows better image quality but higher patient dose.

There are diversity of patients' dose metrics used in CT today and the absence of physicists' agreement about the ideal dose metric that should be used in routine patient dose monitoring [4] [5]. Determining accurate CT dose is more complex than simply using the $\mathrm{CTDI}_{(\mathrm{vol})}$ values reported by the scanner. $\mathrm{CTDI}_{(\mathrm{vol})}$ is dependent on acquisition parameters, as well as calibration phantom diameter, and can result in a significant overestimate or underestimate of the actual dose delivered to the patient because of discrepancies in body size versus calibration phantom size [6].

In-vivo dosimetry using radiochromic films has been used in diagnostic and interventional imaging studies including CT to monitor patient doses. In their study (De Denaro et al., 2007) concluded that, the technique of using in vivo dosimetry measured with radiochromic film appears a promising procedure for improving the assessment of the effective dose to the patient [7].

(Tsalafoutas et al., 2015) after performing point entrance skin dose (ESD) measurements on 64 slice CT scanner in axial and helical modes, conclude that calculating the skin dose distribution in CT examinations is a very challenging task; and a practical approach would be for CT scanners to provide a conservative estimate of the peak skin dose (PSD) using the isocenter entrance surface air kerma (ESAK) value [8].

The PSD is expected to be higher than the ESD in helical CT scanning mode due to overlapping of axial radiation fields at the patient entrance surface. In an effort to verify the clinical dose levels during CT examination of the sinus, we 
are proposing to measure the ESD using XRQA2 films. Using the measured ESD will allow more accurate comparison between scanners and imaging protocols and may serve as dose reference level (DRL) metric in CT imaging. The measured ESD can also be used as an estimator of the patient eye dose in CT examination of the sinus.

The CT exam under consideration must be acquired using the helical acquisition mode with pitch factor less than one is the only criteria that must be used in order to apply the proposed dosimetry method.

The aim of this work was first to study the feasibility of using XRQA2 films as in vivo dosimeter to monitor the ESD during CT examination of the sinus and to measure the PSD. Moreover, to establish a relationship between the measured ESD, PSD and the scanner reported $\mathrm{CTDI}_{(\mathrm{vol})}$, in order to adequately estimate the PSD value based on the scanner archived $\mathrm{CTDI}_{(\mathrm{vol})}$ values for a specific exam and scanner. The method is easy to implement by the institution medical physicist responsible for the annual QA and patient dose evaluation. To the best of our knowledge in vivo measurements of the ESD during CT examination of the sinus using a 64 slices GE CT machine has not been reported yet in the literature.

\section{Materials and Methods}

We have measured the ESD distribution and calculated the PSD. Strips of XRQA2 films were placed at the surface of the female anthropomorphic phantom and later under the patient head during CT scanning of the sinus exam using the routine clinical protocol used at our medical institution. The measured values were compared with the $\mathrm{CTDI}_{(\mathrm{vol})}$ available at the scanner work station. The ratios of $\mathrm{ESD} / \mathrm{CTDI}_{(\mathrm{vol})}$, and $\mathrm{PSD} / \mathrm{CTDI}_{(\mathrm{vol})}$ were calculated.

\subsection{Film Calibration}

The XR-QA2 films (Lot Number: 07311401) were calibrated for air kerma free in air against the reading from PTW pancake calibrated ionization chamber (IC): PTW (SFD-type 34060 with calibration for the relevant X-ray energies and traceable to German National Laboratory (PTB).

The electrometer used was the PTW UNIDOS E REF T10008 (S/N 081096) calibrated according to DIN EN ISO 9001:2008.

Strips of film were placed on top and at the center of $30 \mathrm{~cm} \times 30 \mathrm{~cm}$ PMMA bloc measuring $15 \mathrm{~cm}$ of thickness side by side with the IC. The films were placed in the center of the field of view to avoid the bowtie filter and heel effects. The irradiation was done using the CT scanner X-ray tube in stationary position using the scanner service mode of operation by the system engineer.

The film dosimetry method overall average uncertainty was $8 \%$. Uncertainties were estimated using the method described in [9]. The irradiated film reflective optical density as a function of the scan axial (Z) positions is shown in Figure 1. (a) Along with a picture of the irradiated film (b). 


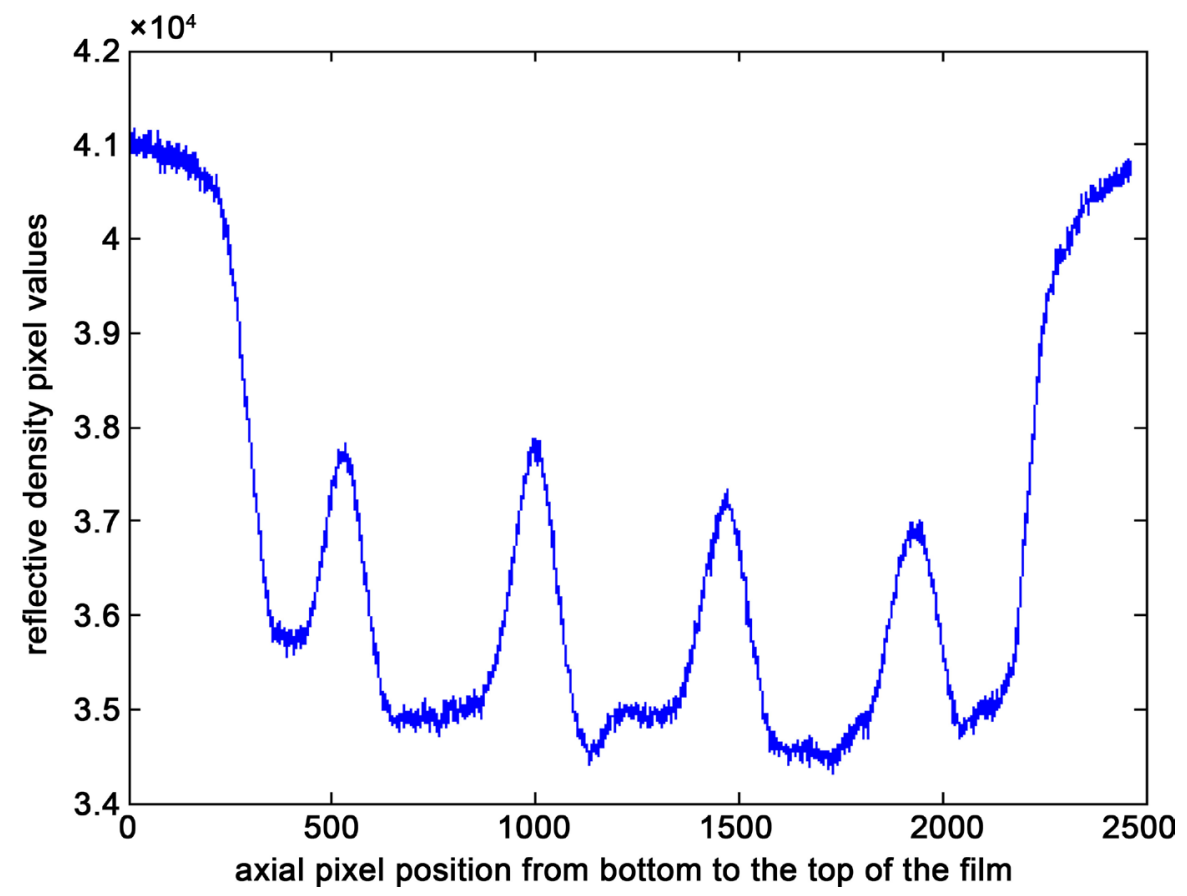

(a)

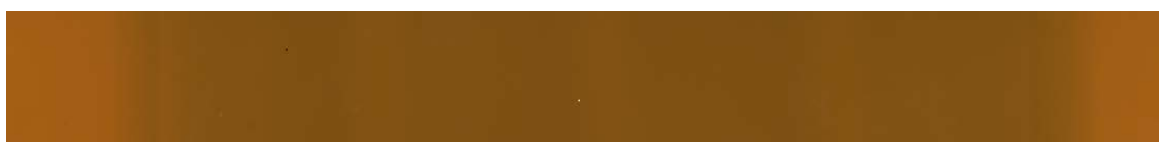

(b)

Figure 1. (a) The beam profile in the axial direction (Z) expressed as optical density values of the scanned film in reflective mode. We notice the sinusoidal shape of the profile which is a characteristic of the helical mode of acquisition with pitch factor of less than one; (b) XR-QA2 film slice placed under the patient head.

\subsection{Irradiated Film Processing}

The films were scanned 24 hours post irradiation as recommended by the (AAPM report No.63, 1998) [10]. The film digitization process was done using the flatbed high resolution scanner Epson 10000XL.

The film resolution was 48 bits for 3 colors mode, 16 bits for each color, red, green and blue (RGB). The film spatial resolution was 72 dots per inch (dpi), the films' digital images were saved in TIFF format with no color correction [11] [12].

The images were then imported to MATLAB software for analysis using in house written routines in MATLAB 7.1 (The Mathworks, Natick, MA). The ESD as function of the films net reflective optical density (NRD) was then obtained as shown in Figure 2.

\subsection{ESD Measured on Phantom}

We have measured The ESD using a heterogeneous anthropomorphic cross sectional dosimetry phantom (CIRS ATOM, model 702-C) adult female head. We have placed 4 pieces of films positioned at 4 locations AP, PA and Right/Left 
Lateral in order to verify the film irradiation uniformity during the CT sinus examination.

\subsection{In-Vivo ESD Measurements}

The films were placed under the patient head rest in order to sample the entrance surface dose in-vivo. We have performed in vivo film irradiation on 23 patients in this study to verify the suitability of the method to be applied in the clinic. The films did not interfere with the diagnostic quality of the examination. Details of the sinus CT imaging protocol are in Table 1. Entrance surface dose (ESD) and the peak surface dose (PSD) where measured in vivo using radiochromic films. The measured ESD and PSD are real estimates of the dose delivered

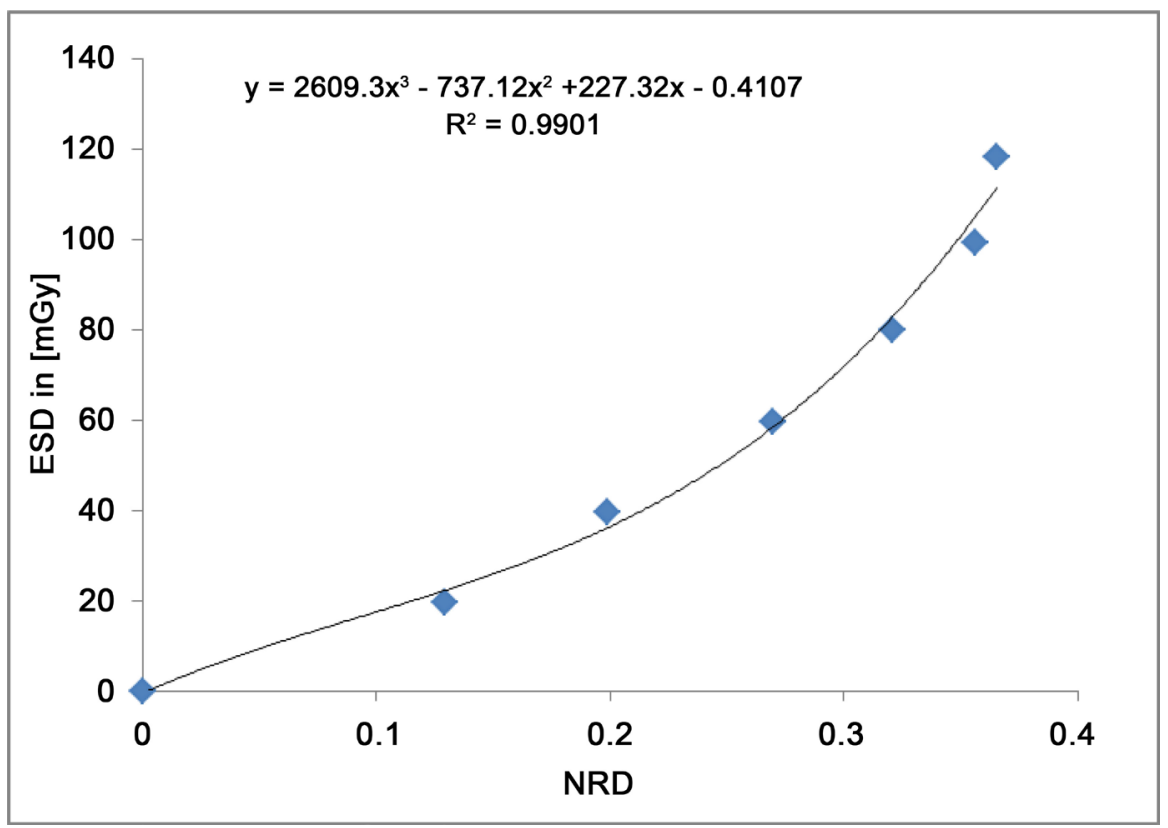

Figure 2. Graph showing the relationship of entrance skin dose (ESD) and XRQA2 film Net Reflective optical Density (NRD).

Table 1. Sinus exam protocol

\begin{tabular}{cc}
\hline Parameter & Value \\
\hline $\mathrm{kVp}$ & 100 \\
$\mathrm{~mA}$ & 150 \\
Spiral Pitch Factor & 0.984 \\
Total Collimation Width & $40 \mathrm{~mm}$ \\
Distance Source to Detector & $949 \mathrm{~mm}$ \\
Distance Source to Patient & $541 \mathrm{~mm}$ \\
Filter Type & MEDIUM FILTER \\
Convolution Kernel & BONEPLUS \\
Scanning Mode & Helical \\
Scanner & GE Light Speed VCT 64 Slices
\end{tabular}


to the patient from a specific scanner and imaging protocol.

In order to control and reduce the errors in dose estimations using this method, we have followed the recommendations of the AAPM report, 1998. [13].

The use of Gafchromic films has been used by medical physicist in developing countries [14] [15]; as well as in developed ones. Such trend proves that radiochromic films are available, affordable and reliable dosimeters that maybe used worldwide [16].

\section{Results}

The objective of this work was to study the implementation of radiochromic films (Gafchromic XR-QA2) as in-vivo dosimetry tool used for patient dosimetry studies in computed tomography (CT) imaging of the sinus.

The scanner displayed $\mathrm{CTDI}_{(\mathrm{vol})}$ average value in [mGy] from the 23 in-vivo measured patients' dose reports was $13.3 \pm 0.1$, the measured ESD at the surface was $11.7 \pm 1.0$ and the peak PSD was $15.7 \pm 1.7$. The calculated ratios were 0.88 and 1.18 for $\mathrm{ESD} \mathrm{CTDI}_{(\mathrm{vol})}$ and $\mathrm{PSD} / \mathrm{CTDI}_{(\mathrm{vol})}$ respectively. The obtained results seem to indicate that the $\mathrm{CTDI}_{(\mathrm{vol})}$ is a conservative estimator for the ESD but it underestimates the PSD by $18 \%$.

The main advantages of using radiochromic films is its ability to measure the entrance surface dose, the surface dose distribution in the axial plane, the peak skin dose and the dose profile in one single exposure. Therefore the utilization of the radiochromic films in routine dosimetry offers a net advantage over the classical ionization chamber. But a cross reference measurement with the ionization chamber is required.

\section{Discussion}

The films neither have the advantage of not interfering with the patient setup nor produce any image artifacts. Such films can be placed at the back side of the patient during the CT procedure [17], or can also be placed facing the X-ray beam entry into the patient body surface during fluoroscopy procedures [18].

(Boivin et al., 2011) have used XR-QA films to measure patient skin doses in vivo during CT examinations while using Rando phantom type. Their measurement covered a dose range from 0 to $200 \mathrm{mGy}$. They measured the entrance surface dose (ESD) using four or eight film strips taped on patients' skin and on Rando phantom. Optical reflectance of the unexposed film piece was subtracted from exposed one to obtain final net reflectance change. They observed that body skin dose variation has a sinusoidal pattern along the scanning axis due to the helical movement of the X-ray tube. Their obtained experimental dose length products (DLP) showed lower values than the ones obtained from the scanner console for the head scans [19].

(De las Heras et al., 2013) related the widely used computed tomography dose index (CTDI) to an actual skin dose measurement. They have measured peak surface dose (PSD) using CTDI head phantom and related the value to the 
CTDI $_{(\mathrm{vol})}$ displayed on the console of CT scanners. They have obtained a scanner specific relationship between the two metrics. The routine head CT exam produced a PSD values between $27-136 \mathrm{mGy}$ among all the tested scanners [20].

$\mathrm{CTDI}_{(\mathrm{vol})}$ was found to overestimates the PSD and eye lens doses in brain perfusion CT scans in a simulation study [21].

The most appropriate application of the method presented in this work is for the medical physicist to examine the surface dose distribution and to evaluate the peak entrance surface dose in CT protocols using large beam width and high pitch factors because the beam width, the pitch factor and the tube start angle are known to affect the surface dose distribution [22].

The disadvantage of the method is the cost of such films since they cannot be reused unlike TLD dosimeter. The dosimetry method is both scanner and protocol specific and the calibration of films against reference ionization chambers must be conducted for each film batch used. On the other hand the main advantage of the method is that it is non-invasive and easy to implement clinically.

The CTDI $($ vol) also is only a dose index is found to be useful parameter that allows the diagnostic medical physicist to perform routine quality assurance on CT scanners, estimate patient doses, and compare doses from different protocols and scanners in the hospital environment. In this study for helical CT sinus examination the CTDI ${ }_{(\mathrm{vol})}$ was a conservative estimator of the exam ESD and by multiplying it with proper conversion factor it will also provide an estimate the PSD.

XRQA2 film can be used as in vivo dosimeter to measure the patient's ESD, PSD and to examine surface dose distribution in computed tomography.

\section{Conclusions}

The film successfully demonstrated its ability to picture the CT radiation dose profile, to measure entrance surface dose (ESD), and to locate and measure the peak surface dose (PSD) during helical CT scan of the Sinus.

The presented method allows easily implemented way to verify and document patient entrance surface dose estimated using a simple conversion factor and $\mathrm{CTDI}_{\text {(vol) }}$ data available with the CT exam dose report archived in the local PACS depository.

Implementation of XRQA2 radiochromic film in-vivo dosimetry does not interfere with the clinical radiological examination and did not cause any imaging artifact.

\section{References}

[1] Hricak, H., Brenner, D.J., Adelstein, J.S., et al. (2011) Managing Radiation Use in Medical Imaging: A Multifaceted Challenge. Radiology, 258, 889-905. https://doi.org/10.1148/radiol.10101157

[2] ACR-AAPM (2012) ACR-AAPM Technical Standard for Diagnostic Medical Physics Performance Monitoring of Computed Tomography (CT) Equipment. (Resolution 34). 
[3] Cody, D.D., et al. (2013) AAPM Medical Physics Practice Guideline 1.a: CT Protocol Management and Review Practice Guideline, Task Group No.225. Journal Applied Clinical Medical Physics, 14, 3-12. https://doi.org/10.1120/jacmp.v14i5.4462

[4] Brenner, D.J., McCollough, C.H. and Orton, C.G. (2006) It Is Time to Retire the Computed Tomography Dose Index (CTDI) for CT Quality Assurance and Dose Optimization? Medical Physics, 33, 1189-1191.

[5] Huda, W. (2013) CT Dose Metrics. Radiology, 267, 964-967. https://doi.org/10.1148/radiol.13122469

[6] Seibert, A.J., Boone, J.M., Wotton-Gorges, S.L. and Lamba, R. (2014) Dose Is Not Always What It Seems: Where Very Misleading Values Can Result from Volume CT Dose Index and Dose Length Product. Journal of the American College of Radiology, 11, 233-237. https://doi.org/10.1016/j.jacr.2013.10.010

[7] De Denaro, M., Bregant, P., Severgnini, M. and de Guarrini, F. (2007) In Vivo Dosimetry for Estimation of Effective Doses in Multislice CT Coronary Angiography. Medical Physics, 34, 3705-3710. https://doi.org/10.1118/1.2766758

[8] Tsalafoutas, I.A., Epistatou, A., Nikoletopoulos, S. and Tspaki, V. (2015) Measuring Skin Dose in CT Examinations under Complex Geometries: Instruments, Methods and Considerations. Physica Medica, 31, 1005-1014.

https://doi.org/10.1016/j.ejmp.2015.08.001

[9] Devic, S., Seuntjens, J., Sham, E., et al. (2005) Precise Radiochromic Film Dosimetry Using a Flat-Bed Document Scanner. Medical Physics, 32, 2245-2253.

https://doi.org/10.1118/1.1929253

[10] Niroomand-Rad, A., et al. (1998) AAPM Report No. 63, Recommendations of Radiation Therapy Committee Task Group No.55. Radiochromic Film Dosimetry. Medical Physics, 25, 2093-2115. https://doi.org/10.1118/1.598407

[11] Martin, C.J., Gentle, D.J., Sookpeng, S., et al. (2011) Application of Gafchromic Film in the Study of Dosimetry Methods in CT Phantoms. Journal of Radiological Protection, 31, 389-409. https://doi.org/10.1088/0952-4746/31/4/001

[12] Rampado, O., Garelli, E., Deagostini, S., et al. (2006) Dose and Energy Dependence of Response of Gafchromic XR-QA Film for Kilovoltage X-Ray Beams. Physics in Medicine and Biology, 51, 2871-2881. https://doi.org/10.1088/0031-9155/51/11/013

[13] Niroomand-Rad, A., et al. (1998) Radiochromic Film Dosimetry: Recommendations of AAPM Radiation Therapy Committee Task Group No. 55. Medical Physics, 25, 2093-2115.

[14] Mourão, A.P., Gonçalves Jr., R.G. and Alonso, T.C. (2014) Dose Profile Variation with Pitch in Head CT Scans using GAFCHROMIC Films. Recent Advances in Biomedical \& Chemical Engineering and Materials Science, 1, 51-54.

[15] Purwaningsih, S., Lubis, L.E., Pawiro, S.A. and Soejoko, D.S. (2016).Measurement of Computed Tomography Dose Profile with Pitch Variation using Gafchromic XR-QA2 and Thermoluminescence Dosimeter (TLD). Journal of Physics. Conference Series, 694, Article ID: 012046. https://doi.org/10.1088/1742-6596/694/1/012046

[16] Lian, C.P.L., Wong, J.H.D., Young, A., Cutajar, D., Petasecca, M., Lerch, M.L.F. and Rosenfeld, A.B. (2013) Measurement of Multislice Computed Tomography Dose Profile with the Dose Magnifying Glass and the MOSkin Radiation Dosimeter. Radiation Measurements, 55, 51-55.

[17] Tsalafoutas, I.A., Tsapaki, V., Triantopoulou, C., et al. (2008) Comparison of Measured and Calculated Skin Doses in CT-Guided Interventional Procedures. American Journal of Roentgenology, 191, 1601-1607. https://doi.org/10.2214/AJR.08.1030 
[18] Soliman, K. and Alenezi, A. (2014) Patient Entrance Surface Dose Measurements using XR-QA2 Gafchromic Films during Micturating Cystourethrography Procedures. Radiation Protection Dosimetry, 158, 170-174.

https://doi.org/10.1093/rpd/nct209

[19] Boivin, J., Tomic, N., Fadlallah, B., Deblois, F. and Devic, S. (2011) Reference Dosimetry during Diagnostic CT Examination using XR-QA Radiochromic Film Model. Medical Physics, 38, 5119-5129. https://doi.org/10.1118/1.3622607

[20] De las Heras, H., Minniti, R., Wilson, S., et al. (2013) Experimental Estimates of Peak Skin Dose and Its Relationship to CT Dose Index using CTDI Head Phantom. Radiation Protection Dosimetry, 157, 536-542. https://doi.org/10.1093/rpd/nct171

[21] Zhang, D., Cagnon, C.H., Villablanca, P.J., et al. (2012) Peak Skin and Eye Lens Radiation Dose from Brain Perfusion CT Based on Monte Carlo Simulation. American Journal of Roentgenology, 198, 412-417. https://doi.org/10.2214/AJR.11.7230

[22] Zhang, D., Savandi, A.S., Demarco, J., et al. (2009) Variability of Surface and Center Position Dose in MDCT: Monte Carlo Simulations using CTDI and Anthropomorphic Phantoms. Medical Physics, 36, 1025-1038.

https://doi.org/10.1118/1.3078053 